\title{
MEASUREMENT OF DEFECT DEPTH IN SHEET METAL BY PULSE THERMOGRAPHY
}

\author{
Soo-Yong Choi ${ }^{1+}$, Jae-Yeon Kim ${ }^{1}$, Min-Jung Park ${ }^{1}$, Jai-Won Park ${ }^{2}$, Won-Sik Lee ${ }^{3}$, Jai-Won Byeon ${ }^{1 *}$ \\ ${ }^{1}$ Department of Materials Science \& Engineering, Seoul National University of Science and Technology, \\ Seoul, Korea \\ ${ }^{2}$ ASEA Aviation College, Seoul, Korea \\ ${ }^{3}$ Advanced Process and Materials R\&D Group Research Institute of Advanced Manufacturing Technology, \\ Korea Institute of Industrial Technology, Incheon, Korea \\ †Presenting Author: sychoi13@seoultech.ac.rk \\ *Corresponding Author: byeonjw@ @eoultech.ac.kr
}

\begin{abstract}
Recently, the demand for sheet metals has been increasing to reduce the size and weight of mechanical parts and structures. Various nondestructive inspection techniques have been studied to evaluate the soundness of sheet metal. The infrared thermography technique has advantages such as portability and large inspection area, but it is difficult to accurately measure the depth of defects in sheet metals. Therefore, to measure the depth of defects, this study has shown the correlation between the standard thermal contrast, defect diameter, defect depth, and thermal conductivity of various materials. In order to investigate the correlation between the standard thermal contrast and the diameter and depth of defects, defects were machined into 1-mm-thick sheet metal. The defect diameters were 2,3 , and $4 \mathrm{~mm}$, and the depths were $0.3,0.4$, and $0.5 \mathrm{~mm}$. The specimens were made of STS304, A1050, and C1020 to investigate the correlation between the standard thermal contrast and thermal conductivity of the materials. The maximum standard thermal contrast of the defects in the STS304 specimen was higher as the defect diameter was larger and the defect depth was shallower. In addition, the lower the thermal conductivity of the material, the higher the maximum standard thermal contrast. This relationship can be represented by an equation, and the depth of the defects estimated by the equation was compared with the actual depth of the defects.
\end{abstract}

KEYWORDS: Sheet metal, Pulse thermography, Defect depth

\section{INTRODUCTION}

Recently, the demand for sheet metals has been increasing to reduce the size and weight of mechanical parts and structures. Defects in sheet metals can cause brittle fractures in the structures and degrade the quality of the mechanical parts. Accordingly, to improve the soundness of sheet metals, various nondestructive tests such as X-ray inspection, magnetic particle tests, and ultrasonic inspection are required in the production process. Among them, the infrared thermography technique is used for monitoring the defects in various fields such as corrosion, fatigue, and welding without any risk factors such as radiation. However, because thermography is represented by two dimensions, it is limited in the sense that it is difficult to measure the depth of the defects. Therefore, studies have been conducted to measure the depth of the defects by investigating the correlation between the temperature distribution and defect depth.[1,2] However, applicability of this method for sheet metals with thicknesses of $1 \mathrm{~mm}$ or less has not been verified. Therefore, in this study, the defect depth was estimated by using an equation that describes the relationship between the parameters derived from thermography and the defects. In order to evaluate the accuracy of the equation, the estimated defect depth was compared with the actual defect depth.

\section{EXPERIMENTAL}

\subsection{PULSE THERMOGRAPHY TECHNIQUE}

Fig. 1 shows a schematic of the pulse thermography technique, which consists of an infrared camera, lamp, and specimen. The infrared camera is located $0.2 \mathrm{~m}$ from the specimen to obtain the specimen's thermal information. The thermography was performed for a sufficient time to detect the defect. The framerate was set to $50 \mathrm{~Hz}$ and the $6 \mathrm{~kJ}$ xenon lamp was located $0.2 \mathrm{~m}$ from the specimen, which was irradiated by the lamp for $2.5 \mathrm{~ms}$ 


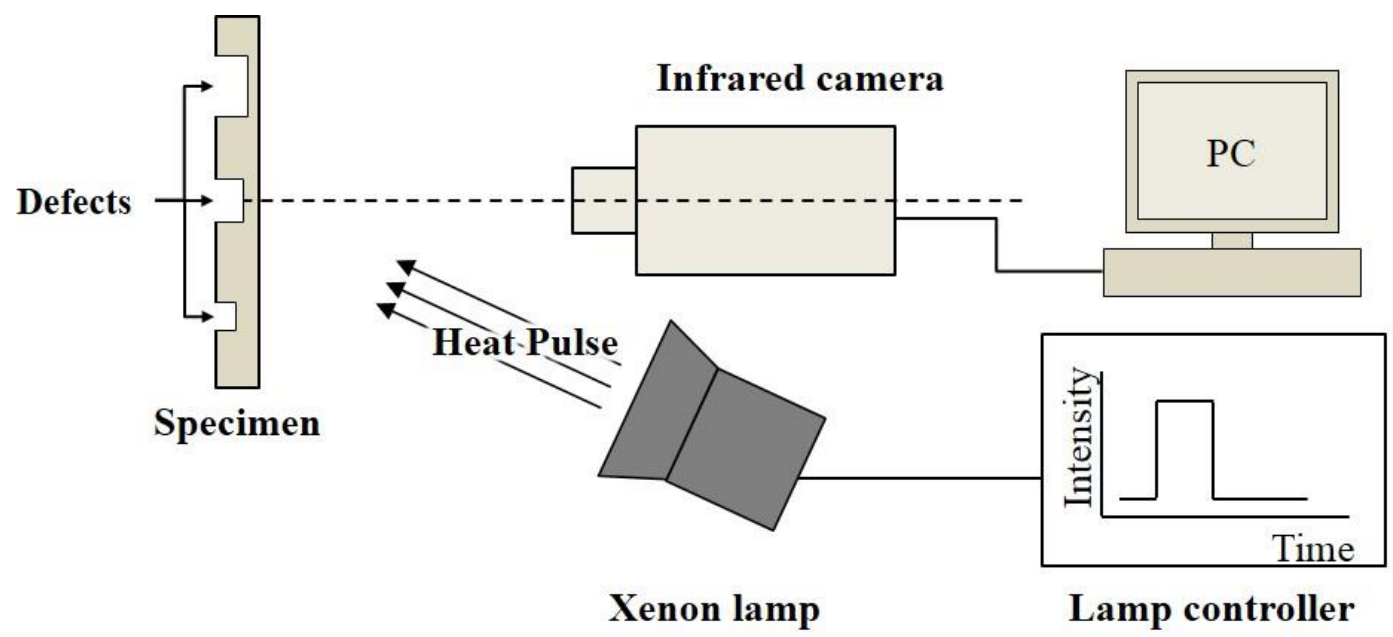

Fig. 1 Schematic of the pulse thermography technique.

\subsection{SPECIMEN}

Fig. 2 shows a schematic of a specimen designed with an artificial defect. In order to investigate the correlation between the depth and diameter of the defects and the thermography, artificial defects were designed with diameters of 2,3 , and $4 \mathrm{~mm}$, and depths of 0.3, 0.4, and $0.5 \mathrm{~mm}$; the specimens were made of STS304, A1050, and C1020. All specimens were coated with graphite paint to exhibit high and homogeneous emissivity.

\subsection{ANALYSIS OF THERMOGRAPHY}

Fig. 3. (a)-(d) show the thermography of STS304 obtained by the pulse thermography technique as a function of time. Figure 3(b) displays the thermography when the specimen was irradiated by the xenon lamp. Figure 3(c) displays the thermography when the specimen was cooling. Figure 3(e) presents the temperature of the sound area of the specimen and defect with a diameter of $4 \mathrm{~mm}$ and depth of $0.3 \mathrm{~mm}$ as a function of time. Defects can be detected by the difference in temperature between the defect and sound area. In addition, the difference in temperature is probably related to the defect size. The difference in temperature can be quantified by the standard thermal contrast $\mathrm{C}(\mathrm{t})$ and can be calculated by equation (1).[3]

$$
C(t)=\left[T_{\text {defect }}(t)-T_{\text {defect }}\left(t_{0}\right)\right] /\left[T_{\text {sound }}(t)-T_{\text {sound }}\left(\mathrm{t}_{0}\right)\right]
$$

where $\mathrm{T}_{\text {defect }}$ is the surface temperature of the defect, $\mathrm{T}_{\text {sound }}$ is the surface temperature of the sound area, $\mathrm{t}_{0}$ is the time before the irradiation, and $t$ is the self-cooling time

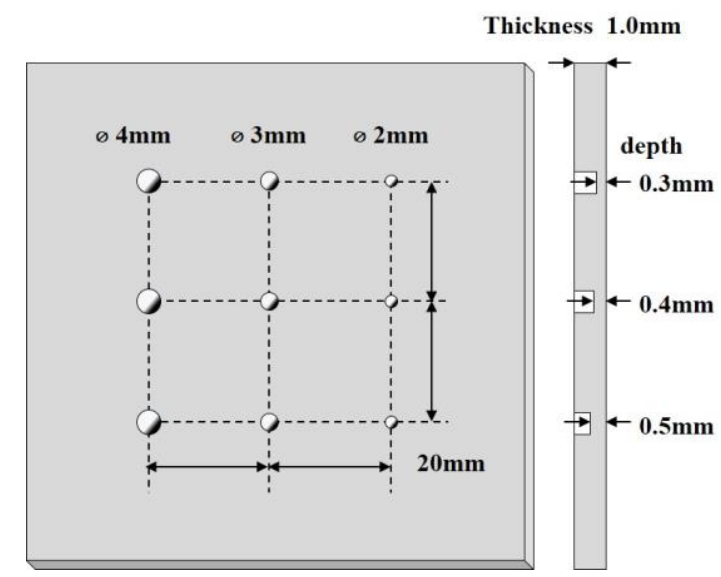

Fig. 2 Schematic of the specimen designed with an artificial defect 


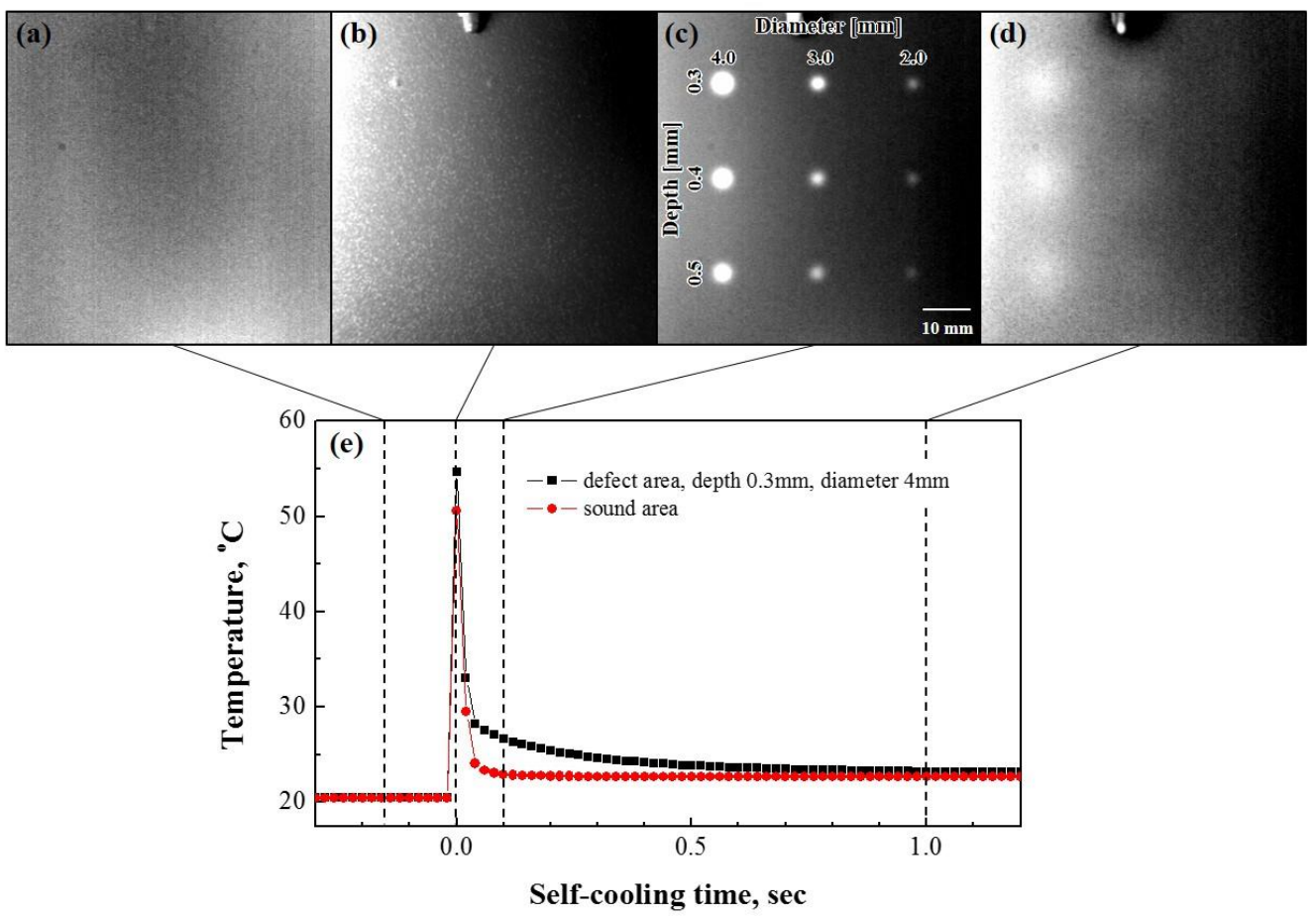

Fig. 33 (a)-(d) Thermography of STS304 as a function of time. (e) Temperature of the sound area and defect with a diameter of $4 \mathrm{~mm}$ and depth of $0.3 \mathrm{~mm}$ as a function of time.

\section{RESULT AND DISCUSSION}

Fig. 4 presents the time dependence of the defects with $C(t)$. All defects exhibit a maximum $C(t)$ at a self-cooling time of $0.1 \mathrm{~s}$. The maximum $\mathrm{C}(\mathrm{t})$ is higher as the diameter of the defect is larger and the depth of the defect is shallower. Based on these qualitative correlations, the correlations between the maximum $\mathrm{C}(\mathrm{t})$ and the depth and diameter of the defects can be represented by equations (2) and (3). These equations were derived from empirical fit models. The maximum $\mathrm{C}(\mathrm{t})$ and defect depth are inversely proportional to each other and can be represented by equation (2).

$$
\mathrm{C}_{\max }=\mathrm{a}_{1} \mathrm{~d}^{-2}+\mathrm{b}_{1}
$$

where $\mathrm{C}_{\max }$ is the maximum $\mathrm{C}(\mathrm{t}), \mathrm{d}$ is the defect depth, and $\mathrm{a}_{1}$ and $\mathrm{b}_{1}$ are constants. The maximum $\mathrm{C}(\mathrm{t})$ and defect diameter are proportional to each other and can be represented by equation (3).

$$
\mathrm{C}_{\max }=\mathrm{a}_{2} \mathrm{D}+\mathrm{b}_{2}
$$

where $\mathrm{D}$ is the defect diameter, and $\mathrm{a}_{2}$ and $\mathrm{b}_{2}$ are constants.
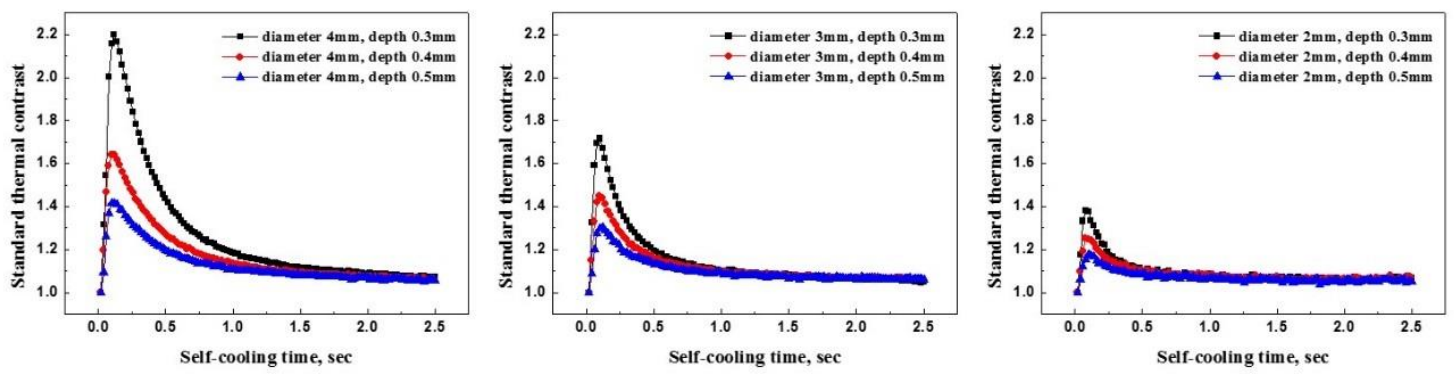

Fig. 4 Dependence of standard thermal contrast with self-cooling time in the STS304 steel specimen. 


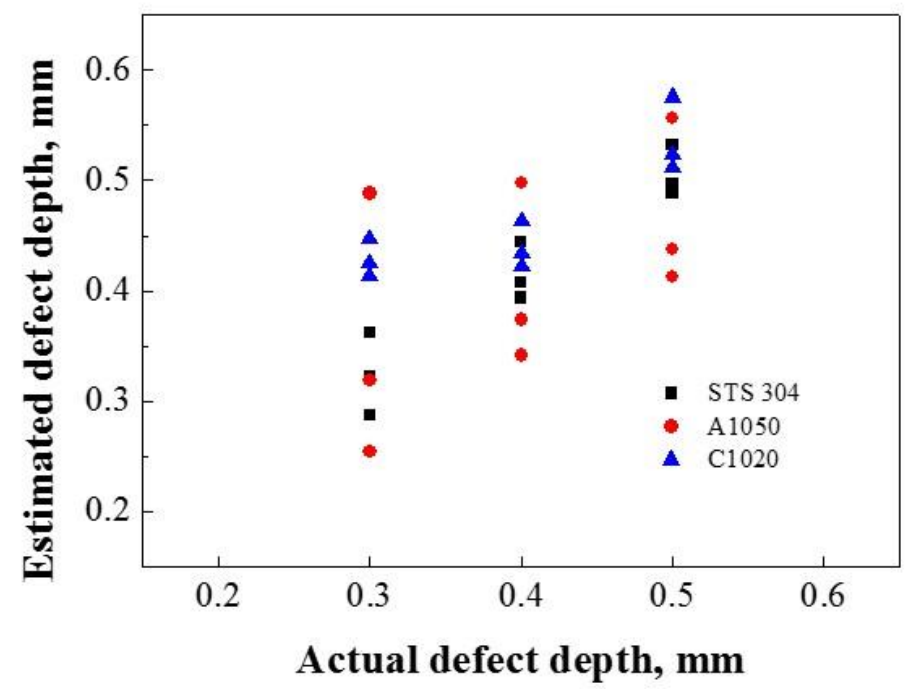

Fig. 5 Comparison of the estimated and actual defect depth.

The surface temperature change is affected by the thermal conductivity of materials in pulse thermography.[4] The correlation between the thermal conductivity and the maximum $\mathrm{C}(\mathrm{t})$ is invers proportional. It is represented by equation (4).

$$
\mathrm{C}_{\max }=\mathrm{a}_{3}(\alpha)^{-1 / 2}+\mathrm{b}_{3}
$$

where $\alpha$ is the thermal conductivity of the specimen, and $a_{3}$ and $b_{3}$ are constants. Assuming that equations (2), (3), and (4) are independent, the defect depth $\mathrm{d}$ can be represented by equation (5).

$$
\mathrm{d}=\left[\mathrm{kD} /\left(\mathrm{C}_{\max }-1\right)(\alpha)^{1 / 2}\right]^{1 / 2}
$$

where $\mathrm{k}$ is a constant. Figure 5 shows the comparison of the estimated defect depth using equation (5) and the actual defect depth. In that graph, the results of STS304 and A1050 appear highly linear, while C1020 appears with a low linearity. The percentage error between the estimated and actual defect depth is $6.25 \%$ in STS 304, 19.01\% in A1050, and 20.07\% in C1020, and the lower the thermal conductivity, the lower the percentage error. In Fig. 6, it is revealed that the higher the maximum $\mathrm{C}(\mathrm{t})$, the lower the percentage error.

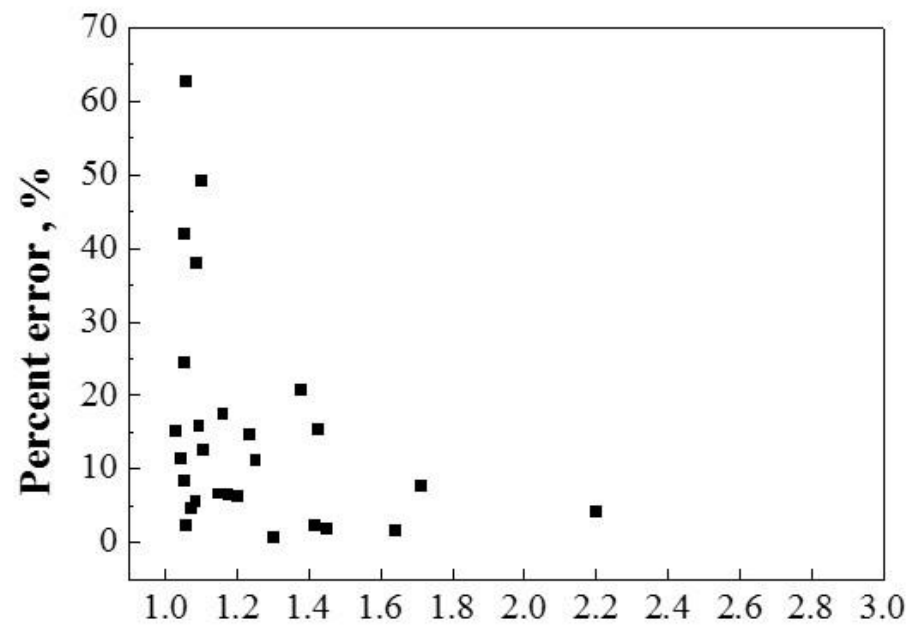

Maximum standard thermal contrast

Fig. 6 Dependence of percentage error on standard thermal contrast. 


\section{CONCLUSIONS}

The depth of defects can be estimated by pulse thermography in sheet metal. The correlation between the maximum $\mathrm{C}(\mathrm{t})$, defect depth, defect diameter, and thermal conductivity can be represented by quantitative equations, using which the depth of the defects was estimated. The percentage error between the estimated and actual depth of defects was $6.25 \%$ in STS 304, $19.01 \%$ in A1050, and 20.07\% in C1020. It was revealed that the lower the thermal conductivity, the lower the percentage error.

\section{ACKNOWLEDGEMENT}

This work was supported by the Technology Innovation Program (No. 10049237, Development of rapid mold manufacturing technology for mass customized medical devices with SLS hybrid 3D printing technology) funded By the Ministry of Trade, industry \& Energy(MI, Korea)"

\section{REFERENCES}

[1] Olga Wysocka-Fotek, Reconstruction of size and depth of simulated defects in austenitic steel plate using pulsed infrared thermography, Infrared Physics \& Technology 55 (2012) 363-367

[2] J. G. Sun, Analysis of Pulsed Thermography Methods for Defect Depth Prediction, Journal of Heat Transfer Vol.128 (2006) $331-338$

[3] X.P.V. Maldague, Theory and Practice of Infrared Technology for Nondestructive Testing, Willey-Interscience, New York, 2001, pp. 198-199.

[4] W. J. Parker, Flash Method of Determining Thermal Diffusivity, Heat Capacity, and Thermal Conductivity, J. Appl. Phys. 32 (1961), 1679-1684. 